\title{
Maltrato infantil $y$ trastornos mentales delincuentes juveniles: Una revisión sistemática
}

\section{Child abuse and mental disorders in juvenile delinquents: A systematic review}

\author{
Viviana Morales-Toro ${ }^{1}$, Alejandro Guillén-Riquelme ${ }^{2}$, Raúl Quevedo-Blasco ${ }^{3}$ \\ ${ }^{1}$ Universidad de Granada. vivianamorales@correo.ugr.es \\ ${ }^{2}$ Centro de Investigación Mente, Cerebro y Comportamiento (CIMCYC). Universidad de Granada. agr@ugr.es \\ ${ }^{3}$ Centro de Investigación Mente, Cerebro y Comportamiento (CIMCYC). Universidad de Granada. rquevedo@ugr.es
}

Recibido: 30/9/2019

Aceptado: 4/12/2019

Copyright (C)

Facultad de CC. de la Educación y Deporte. Universidad de Vigo

\section{Dirección de contacto:}

Dr. Raúl Quevedo-Blasco.

Facultad de Psicología, Universidad de Granada.

Campus Universitario de Cartuja, s/n. 18011 GRANADA (ESPAÑA).

\section{Resumen}

La delincuencia y su relación con los traumas y trastornos es un tema de gran interés. El objetivo principal de esta revisión es analizar la posible asociación entre experiencias traumáticas en la infancia y el desarrollo de determinados trastornos, en adolescentes vinculados al sistema judicial. Para ello, se realizó una búsqueda bibliográfica en Web of Science (colección principal), Scopus, Medline, Psycinfo, Cochrane, Teseo, Psicodoc y Scielo, de documentos publicados hasta el año 2018 (incluido). Un total de 43 documentos cumplieron con los criterios de inclusión y por tanto fueron analizados en la presente revisión. En estos resultados se observó que el trastorno del humor (afectivo) suele ser el más frecuente en esta población y dentro de las experiencias traumáticas primaron el abuso sexual y haber sufrido diferentes tipos de experiencias traumáticas. Se observó que el trastorno del humor estuvo mayoritariamente vinculado con el abuso sexual, el TEPT se asoció con diferentes experiencias traumáticas y el trastorno de personalidad con experiencias traumáticas y abuso físico. Parece que existe relación entre el trauma infantil y el desarrollo de trastornos mentales.

\section{Palabras Clave}

Trastorno de Estrés Postraumático, Trastorno del Humor, Trastorno de Personalidad, Abuso, Delincuencia

\section{Abstract}

The delinquency and its relationship with traumas and disorders is a topic of great interest. The main of this review is to analyse the possible association between traumatic experiences in childhood and the development of disorders in juveniles linked with the judicial system. For this, a bibliographic search was carried out in Web of Science (main collection), Scopus, Medline, Psycinfo, Cochrane, Teseo, Psicodoc and Scielo, of documents published until 2018 (included). A total of 43 documents met the inclusion criteria and therefore analysed in the present review. These results show that mood disorder (affective) is more frequent in this population and in the context of traumatic experiences, sexual abuse and different types of traumatic experiences. Within the associations, it was found that mood disorder was 
mostly linked to sexual abuse, while PTSD was associated with different traumatic experiences and personality disorder to the different traumatic experiences and physical abuse. There seems to be relation between childhood trauma and the development of mental disorders.

\section{Key Words}

Post-traumatic Stress Disorder, Mood Disorder, Personality Disorder, Abuse, Delinquency

\section{INTRODUCCIÓN}

La Organización Mundial de la Salud (OMS) define la adolescencia como un período de crecimiento y desarrollo del ser humano que transcurre entre la niñez y la edad adulta, abarcando entre los 10 y los 19 años (OMS, 2015). Según el Instituto Nacional de Estadística (INE, 2017), se ha encontrado que, en 2017, un total de 13.643 adolescentes con edades entre los 14 y 17 años fueron condenados por haber cometido algún tipo de delito, de los cuales 10.819 fueron hombres y 2.824 mujeres. Las edades dentro de ese rango con mayor proporción de delincuentes fueron los 17 y 16 años. Aunque el porcentaje de delitos sea mayor en los hombres (Yugueros-García, 2013), parece que las tasas de arresto en mujeres están aumentando (Zahn, Hawkins, Chiancone y Whitworth, 2008).

La OMS cataloga como maltrato infantil a todo abuso y desatención que dañen o puedan dañar a la salud, desarrollo o dignidad del niño, o pongan en peligro su supervivencia (OMS, 2016). Este puede tener graves consecuencias psicológicas, físicas y jurídicas. Por ello, se hace preciso analizar el maltrato infantil o diferentes experiencias traumáticas como predictor de la conducta delictiva en la adolescencia (Baglivio et al., 2014; Cerezo y Vera, 2004; Fox, Perez, Cass, Baglivio y Epps, 2015; Wolff, Baglivio y Piquero, 2017), coincidiendo esto último con un estudio donde la muestra de sujetos estaba privada de libertad (Vallejos y Bertone, 2016).

Dentro del maltrato infantil se ha encontrado que el más frecuente es el abuso emocional, seguido del físico, el abuso sexual y la negligencia (Dias, Sales, Mooren, Mota-Cardoso y Kleber, 2017; Ulloa-Flores y Navarro-Machuca, 2011). En varones y mujeres privadas de libertad, también se encontraron resultados similares a los expuestos en la población no delincuente, anteriormente comentada, en muestras de diferentes países (Caravaca-Sánchez, Ignatyev y Mundt, 2018; Schimmenti, Di Carlo, Passanisi y Caretti, 2015; Vallejos, Bertone y Muniello, 2014). En otro estudio que no evaluó el abuso emocional, se encontraron mayores tasas de negligencia, abuso físico y sexual (Clements-Nolle, Larson, Buttar y Dermid-Gray, 2017).

Es preciso tener en cuenta que en ocasiones los jóvenes detenidos, tanto hombres como mujeres, muestran trastornos mentales (Fazel, Doll y Långström, 2008; Teplin, Abram, McClelland, Washburn y Pikus, 2005), además de otros factores asociados (Alarcón, Pérez-Luco, Wenger, Salvo y Chesta, 2018; Férriz, Sobral y Gómez-Fraguela, 2018; Llorca-Mestre, Malonda-Vidal y Samper-García, 2017). En una muestra de delincuentes sexuales, se encontró que uno de los trastornos más frecuentes fue el afectivo (Becerra-García y García-León, 2012). El abuso infantil tiene repercusión en trastornos como la depresión, siendo el doble para los reclusos supervivientes de todo tipo de abuso en la infancia que para aquellos que no habían estado expuestos (Caravaca 
et al., 2018). La exposición severa a múltiples tipos de traumas, es cada vez más frecuente en mujeres delincuentes, siendo las tasas del trastorno de estrés postraumático (TEPT) superiores al 30\% (Foy, Ritchie y Conway, 2012). En una muestra de hombres delincuentes también se probó que la exposición a múltiples tipos de trauma, se vinculó a mayores niveles de TEPT (Stimmel, Cruise, Ford y Weiss, 2014). Otro de los trastornos con los que se ha vinculado a la población delincuente con algún tipo de maltrato en la infancia, es el trastorno de personalidad (Vallejos et al., 2014; Zhang y Zheng, 2018).

Por todo lo visto, el objetivo de esta investigación es explorar la posible relación en la población adolescente delincuente (estén detenidos en un centro de internamiento o cumpliendo condena de alguna otra forma), los delincuentes juveniles, entre el maltrato infantil y los trastornos del humor (afectivos), los trastornos específicos de la personalidad, o el TEPT. Más concretamente se desglosará en: a) examinar el tipo de trastorno que más se da en esta población de manera general y/o teniendo en cuenta los diferentes subtipos de cada trastorno elegido, b) detectar el tipo de abuso que más suelen sufrir en la infancia los delincuentes juveniles, y c) asociar el trastorno al tipo de abuso que suele provocarlo con más frecuencia.

\section{MÉTODO}

\subsection{Diseño y procedimiento}

Se ha realizado una Revisión Sistemática (RS), siguiendo las pautas de PeresteloPérez (2013) y las recomendaciones de la guía PRISMA (Urrútia y Bonfill, 2010). Desde el 11 de marzo al 24 de mayo de 2019, se ha realizado una búsqueda exhaustiva de "artículos", "revisiones" y "tesis" publicados hasta 2018 (incluido). Para ello se han usado las siguientes bases de datos: Scopus, Web of Science (en la colección principal), PsycInfo (a través de Proquest), Medline (a través de Web of Science), Psicodoc, Teseo, Scielo (a través de Web of Science) y Cochrane. Los términos que se han utilizado en la búsqueda han sido: <<"Child* abuse*" OR "physical* abuse*" OR "sexual* abuse*" OR "psychologic* abuse*" OR "emotional* abuse*" OR trauma* OR advers* OR maltreat* AND "personality disorder*" OR psychopath* OR paranoid OR schizoid OR antisocial OR disocia* OR borderline OR impulsive OR histrionic OR anancastic OR avoidant OR dependent* OR "PTSD" OR "post-traumatic stress disorder" OR "mood disorder*" OR affective OR bipolar OR depressi* OR dysthymia OR cyclothymia OR mania AND juvenile OR adolescen* OR teen* AND offend* OR delinquen* OR felon OR *prison* OR correctional OR reformatory OR "approved school" NOT adult*>>. Estos términos se usaron en inglés y/o español y se buscaron en "título, resumen y palabras clave" (con los truncadores y boleanos detallados), adaptando la fórmula a las bases de datos utilizadas. Asimismo, los resultados se restringieron a artículos, revisiones y tesis. Los idiomas de los documentos que se han filtrado han sido el inglés y español. 


\subsection{Criterios de elegibilidad}

\subsubsection{Criterios de inclusión}

Población delincuente (privada de libertad, libertad vigilada o alguna otra forma de cumplimiento de condena), que haya sufrido algún tipo de maltrato o abuso en la infancia, y que esto le haya provocado algún tipo de trastorno del humor (afectivo), de personalidad o TEPT. Se tendrá en cuenta la edad, personas adolescentes de entre $10 \mathrm{y}$ 19 años. Se incluyen ambos sexos y la población se recabará a nivel mundial. Además, se tendrá en cuenta cualquier estudio (empírico, aleatorizado, experimental, cuasiexperimental y observacional) con datos pretratamiento.

\subsubsection{Criterios de exclusión}

Aquella población no privada de libertad, que no se encuentre en libertad vigilada o no esté cumpliendo o haya cumplido condena por trámites legales. Que sobrepasen el margen de edad marcado, tanto por debajo (menores de 10 años), como por arriba (mayores de 19 años) y aquellos que no hayan sufrido maltrato en la infancia o esto no le haya acarreado un trastorno de los especificados en los criterios de inclusión.

\subsection{Riesgo de sesgo: calidad metodológica}

La calidad metodológica de los estudios incluidos en esta RS, se realizó usando las diferentes escalas de calidad STROBE (von Elm et al., 2014) y SQUIRE Guidelines 2.0 (Ogrinc et al., 2015), adaptándolas al formato de los documentos y a las características de los mismos. Con esto se pretendía clasificar los artículos en cinco categorías de calidad: baja, media baja, media, media alta y alta, aunque en este caso se ha verificado que todos están incluidos en la categoría media-alta, por lo que no se procedió a la eliminación de ningún documento por la calidad.

\subsection{Fiabilidad de la codificación}

Las investigaciones fueron codificadas en las variables analizadas en esta RS por dos codificadores con experiencia en la codificación que se repartieron a partes más o menos iguales (21 y 22) los estudios. Una semana después de finalizar la codificación original, los codificadores volvieron a codificar 10 artículos que ya habían codificado previamente y otros 10 del otro codificador. Siguiendo la técnica de análisis de contenido categorial y aplicando la solución estadística de Fariña, Arce y Novo (2002), la codificación fue evaluada en términos de acuerdo inter-codificadores e intracodificadores con la kappa verdadera ( $\kappa)$, esto es, corregido el acuerdo con la verificación de la exacta correspondencia en la codificación. De este modo se registraba la codificación como 1 acuerdo si se codificaba igual (verificación de la correspondencia; por ejemplo, si ambos codificadores, o en el momento 1 y 2, registraban el trastorno de estrés postraumático como presente en el mismo punto del artículo); 1 desacuerdo si un codificador, o en un momento, registraba una categoría y otro codificador, o en otro momento, no la registraba (por ejemplo, si un codificador, o en el momento 1, registraba trastorno de estrés postraumático y el otro codificador, o en 
el momento 2, no lo registraba); y 2 desacuerdos si ambos codificadores, o en ambos momentos, registraban la misma categoría pero de modo diferente (verificación de la falta de correspondencia; por ejemplo, si un codificador, o en el momento 1, registraba la categoría trastorno de estrés postraumático en un lugar del artículo y el otro, o en el momento, lo registraba en otro lugar del artículo). Los resultados de la concordancia verdadera mostraron una concordancia inter- e intra-evaluador en todas las categorías muy buena $(\kappa>, 81)$. Además, uno de los codificadores también mostró un muy elevado grado de acuerdo (concordancia) con otro codificador en el análisis de categorías en otra RS, concordancia inter-contextos. Cotejada la consistencia inter- e intra-evaluador e inter-contextos, la codificación es fiable (otro codificador entrenado en la técnica de codificación hubiera creado un base de datos similar; Monteiro, Vázquez, Seijo y Arce, 2018).

\section{RESULTADOS}

\subsection{Selección de estudios}

De los 1.975 documentos obtenidos en las diferentes bases de datos, 579 fueron eliminados por duplicados. De los 1.396 restantes, se verificaron por "título y/o resumen", descartando 1.202 documentos que no cumplían con los criterios de inclusión y exclusión y 151 fueron excluidos tras una revisión del texto completo. La muestra final consistió en 43 documentos (39 artículos y 4 tesis). En la Figura 1 se puede ver más claramente el diagrama de flujo seguido. 


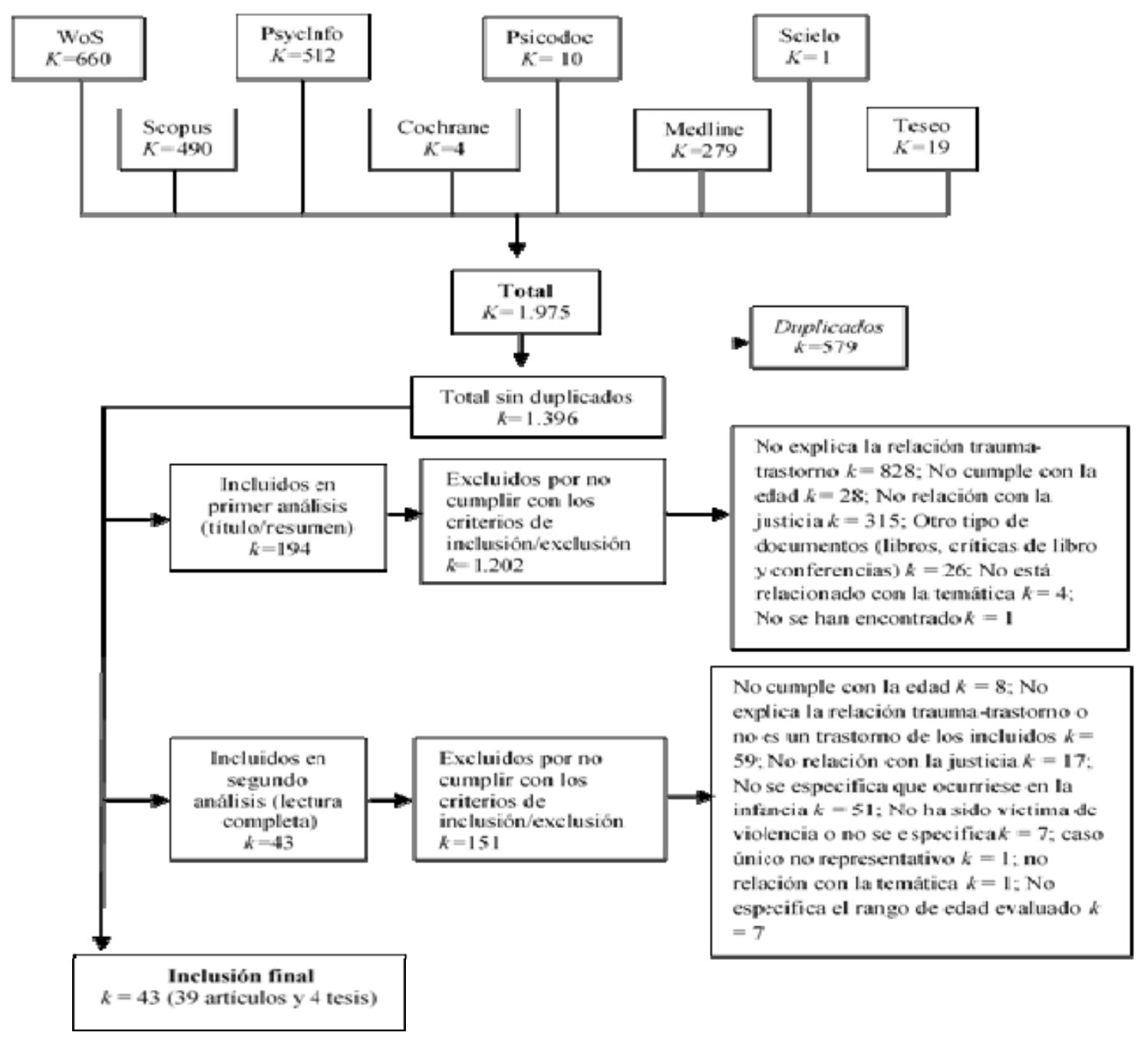

Figura 1. Resultados de la búsqueda y selección de estudios (diagrama de flujo)

\subsection{Características de los estudios}

En este caso las características más relevantes de los documentos que se han incluido en esta RS (referencias con asterisco), se han especificado en la Tabla 1 (características de la muestra, variables y resultados principales), la cual ha sido ordenada según el orden alfabético de los autores de los estudios. 


\begin{tabular}{|c|c|c|c|c|c|}
\hline Autores (año)* & Muestra & País & Variables (trauma) & Variables (trastorno) & Resultados principales \\
\hline Aebi et al. (2015) & $\begin{array}{l}N=260 \text { chicos } \\
\text { Edad media }=16,5 \\
\quad \text { Detenidos }\end{array}$ & Austria & NM, EP, EPS & Trastornos afectivos & $\begin{array}{l}\text { Los varones en las clases EP y EPS, en comparación con los } \\
\text { NM, tenían con mayor frecuencia TDAH, trastorno de conducta, } \\
\text { trastornos afectivos y de ansiedad, pero no se asoció al consumo } \\
\text { de drogas. }\end{array}$ \\
\hline Ariga et al. (2008) & $\begin{array}{c}N=64 \text { chicas } \\
\text { Edad media }=17,2 \\
\quad \text { Detenidas }\end{array}$ & Japón & $\begin{array}{l}\text { Múltiples eventos traumáticos (abuso } \\
\text { sexual, víctima de violencia, noticias } \\
\text { traumáticas...) }\end{array}$ & TEPT & $\begin{array}{l}\text { Experimentar eventos traumáticos (siendo el abuso sexual el más } \\
\text { frecuente), es grave y frecuente en las delincuentes. Esto se } \\
\text { relacionó con una mayor prevalencia de TEPT. Las chicas con } \\
\text { TEPT mostraron mayor comorbilidad psiquiátrica, incluida la } \\
\text { depresión y ansiedad. }\end{array}$ \\
\hline Bielas et al. (2016) & $\begin{array}{l}N=130 \text { chicos } \\
\text { Edad media }=16,84 \\
\quad \text { Detenidos }\end{array}$ & Suiza & $\begin{array}{l}\text { ETI (enfermedad mental, } \\
\text { abuso sexual...) }\end{array}$ & TEPT y depresión & $\begin{array}{l}\text { La puntuación total de ETI predijo el TEPT, el trastorno de } \\
\text { ansiedad, la depresión y tendencias suicidas. Estos resultados } \\
\text { seguían siendo significativos cuando se tenían en cuenta los } \\
\text { síntomas de irritabilidad. }\end{array}$ \\
\hline $\begin{array}{l}\text { Boonmann et al. } \\
\text { (2016) }\end{array}$ & $\begin{array}{c}N=44 \text { (JSO) } \\
\text { Y } 44 \text { (No JSO), ambos chicos } \\
\text { Edad media: JSO }=17,0 \\
\text { No JSO }=17,7 \\
\text { Detenidos }\end{array}$ & Países Bajos & Abuso emocional & $\begin{array}{c}\text { Problemas } \\
\text { depresivos/ansiosos }\end{array}$ & $\begin{array}{l}\text { En los JSO encontraron correlaciones medias entre abuso } \\
\text { emocional y problemas depresivos-ansiosos y la escala de } \\
\text { experiencias traumáticas. En los No JSO las correlaciones eran } \\
\text { entre abuso emocional y escala de experiencias traumáticas, y } \\
\text { entre abandono emocional e ideación suicida. }\end{array}$ \\
\hline $\begin{array}{l}\text { Coleman y Stewart } \\
\text { (2010) }\end{array}$ & $\begin{array}{c}N=398(285 \text { chicos y } 113 \text { chicas }) \\
\text { Edad media }=15,4 \\
\text { Detenidos } / \text { as }\end{array}$ & EE.UU. (Florida) & Abuso (físico y sexual) & Depresión & $\begin{array}{l}\text { Se encontró relación entre el abuso sexual y la depresión, pero } \\
\text { no entre el abuso físico y la depresión. }\end{array}$ \\
\hline $\begin{array}{l}\text { Daversa y Knight } \\
\quad(2007)\end{array}$ & $\begin{array}{c}N=329 \text { chicos } / \text { as } \\
\text { Edad media }=15,17 \\
\quad \text { Detenidos } / \text { as }\end{array}$ & $\begin{array}{l}\text { EE.UU. (Maine, } \\
\text { Massachusetts, } \\
\text { Minesota y } \\
\text { Virginia) } \\
\end{array}$ & Abuso físico y emocional & Psicopatía & $\begin{array}{l}\text { El abuso emocional y físico se consideraron antecedentes } \\
\text { significativos del desarrollo, para predecir la psicopatía. }\end{array}$ \\
\hline Day et al. (2013) & $\begin{array}{l}N=112(68 \text { chicos y } 44 \text { chicas }) \\
\text { Edad media }=16.8 \\
\text { Detenidos } / \text { as y cumpliendo } \\
\text { condena }\end{array}$ & $\begin{array}{l}\text { Canadá (Gran } \\
\text { Toronto) }\end{array}$ & Abuso emocional y físico & Depresión & $\begin{array}{l}\text { Tanto el abuso físico infantil como el emocional se asociaron } \\
\text { con una mayor depresión, que a su vez se asoció a una mayor } \\
\text { probabilidad de victimización. }\end{array}$ \\
\hline Dierkhising et al. (2013) & $\begin{array}{l}N=658(303 \text { chicos y } 355 \text { chicas }) \\
\quad \text { Edad media }=15,7 \\
\text { Detenidos } / \text { as y cumpliendo condena }\end{array}$ & -- & $\begin{array}{l}\text { Diferentes experiencias traumáticas en } \\
\text { la infancia, maltrato emocional y físico }\end{array}$ & $\begin{array}{l}\text { TEPT y síntomas de } \\
\text { internalización } \\
\text { (pensamiento } \\
\text { abandono/ deprimido, } \\
\text { ansioso/ depresivo) }\end{array}$ & $\begin{array}{l}\text { Para hombres y mujeres se hallaron diferencias respecto a la } \\
\text { edad de inicio del trauma y problemas de salud mental, para } \\
\text { mujeres el inicio temprano del trauma se asoció a un TEPT total } \\
\text { más alto y con problemas de internalización. Para los hombres, } \\
\text { sin embargo, la relación fue asociada a problemas de } \\
\text { externalización. }\end{array}$ \\
\hline $\begin{array}{l}\text { Dixon, Howie y } \\
\text { Starling (2004) }\end{array}$ & $\begin{array}{c}N=100 \text { chicas grupo clínico; } 100 \\
\text { chicasgrupo control } \\
\text { Edad media }=16,5 \\
\text { Detenidas }\end{array}$ & Australia & $\begin{array}{l}\text { Testigos de crimen violento, noticias } \\
\text { traumáticas, violencia doméstica, } \\
\text { abuso sexual y abuso físico }\end{array}$ & Depresión y TEPT & $\begin{array}{l}\text { Los resultados mostraron relación entre la exposición al trauma, } \\
\text { la salud mental (TEPT, depresión y abuso de sustancias) y } \\
\text { comportamiento antisocial. }\end{array}$ \\
\hline
\end{tabular}




\begin{tabular}{|c|c|c|c|c|c|}
\hline $\begin{array}{l}\text { Dixon, Howie y } \\
\text { Starling (2005) }\end{array}$ & $\begin{array}{l}N=37 \text { chicas } \\
\text { Edad media }=16,5 \\
\quad \text { Detenidas }\end{array}$ & Australia & $\begin{array}{l}\text { Trauma (abuso sexual; confrontación } \\
\text { con noticias traumáticas; víctima de } \\
\text { crimen violento y otros) }\end{array}$ & TEPT & $\begin{array}{l}\text { El abuso sexual fue el mayor predictor de TEPT, seguido de } \\
\text { haberse presenciado o confrontado un trauma que involucra a un } \\
\text { miembro de la familia, o ser víctima de un trauma en el que } \\
\text { temió por su vida. }\end{array}$ \\
\hline $\begin{array}{l}\text { Farina, Holzer, DeLisi } \\
\text { y Vaughn (2018) }\end{array}$ & $\begin{array}{c}\text { Pensilvania: } N=153 \text { chicos y } 101 \\
\text { chicas } \\
\text { Edad media }=16,24 \\
\text { Misouri: } N=629 \text { chicos y } 94 \text { chicas } \\
\text { Edad media }=15,49 \\
\text { Detenidos/as }\end{array}$ & $\begin{array}{l}\text { EE.UU. } \\
\text { (Pensilvania y } \\
\text { Misouri) }\end{array}$ & $\begin{array}{l}\text { Abuso (físico y emocional), estrés } \\
\text { familiar }\end{array}$ & Psicopatía & $\begin{array}{l}\text { En ambas muestras, además, el trauma infantil (abuso físico, } \\
\text { emocional y estrés familiar) se asoció con características } \\
\text { psicopáticas entre las niñas. Para los niños el trauma sólo fue } \\
\text { predictivo de psicopatía en la muestra de Misouri. Aunque la } \\
\text { psicopatía se asoció con abuso físico y emocional en ambos } \\
\text { sexos, fue mayor en los niños. }\end{array}$ \\
\hline Farris (2007) & $\begin{array}{l}N=614 \text { chicos } \\
\text { Edad }=10 \text { a } 19 \\
\text { Detenidos }\end{array}$ & $\begin{array}{l}\text { EE.UU. } \\
\text { (Alabama) }\end{array}$ & Abuso sexual & Depresión & $\begin{array}{l}\text { Se encontraron niveles más altos de depresión en individuos con } \\
\text { inicio de abuso sexual antes de los } 12 \text { años, en comparación con } \\
\text { aquellos sin antecedentes de abuso sexual. }\end{array}$ \\
\hline Gaete et al. (2018) & $\begin{array}{c}N=935 \text { chicos } \\
\text { Edad media }=17,6 \\
\text { Detenidos }\end{array}$ & Chile & Eventos traumáticos en la infancia & Depresión o distimia & $\begin{array}{l}\text { Las variables asociadas con la depresión o distimia fueron años } \\
\text { de exposición a maltrato infantil, vivir en las calles, } \\
\text { comportamiento delincuente temprano y trastorno de ansiedad. }\end{array}$ \\
\hline $\begin{array}{l}\text { Ghazali, } \\
\text { Chen y } \\
\text { Aziz } \\
(2018)\end{array}$ & $\begin{array}{c}N=207 \text { grupo clínico }(158 \text { chicos y } \\
169 \text { chicas }) ; 120 \text { grupo control } \\
\text { Edad media }=15,3 \\
\text { Detenidos } / \text { as }\end{array}$ & $\begin{array}{l}\text { Malasia } \\
\text { (Sarawak) }\end{array}$ & $\begin{array}{l}\text { Diferentes experiencias traumáticas en } \\
\text { la infancia (maltrato, abuso sexual, } \\
\text { agresión severa, negligencia y } \\
\text { victimización familiar) }\end{array}$ & $\begin{array}{l}\text { TEPT y síntomas } \\
\text { depresivos }\end{array}$ & $\begin{array}{l}\text { Los síntomas depresivos fueron más frecuentes que el TEPT. } \\
\text { Los adolescentes delincuentes altamente victimizados tuvieron } \\
\text { un riesgo mayor de desarrollar síntomas psiquiátricos. }\end{array}$ \\
\hline $\begin{array}{l}\text { Goodkind, Ruffolo, } \\
\text { Bybee y Sarri (2009) }\end{array}$ & $\begin{array}{l}\quad N=186 \text { chicas } \\
\text { Edad media }=15,89 \\
\text { Cumpliendo condena }\end{array}$ & -- & $\begin{array}{l}\text { Experiencias de abuso (físico, } \\
\text { emocional y sexual) }\end{array}$ & Depresión & $\begin{array}{l}\text { Los eventos negativos y abuso (físico y emocional), se asociaron } \\
\text { a estrategias de afrontamiento negativas, lo cual fue un predictor } \\
\text { indirecto de síntomas depresivos, siendo más fuerte la relación } \\
\text { para eventos negativos que para abuso. El abuso sexual no se } \\
\text { relacionó a los síntomas depresivos. }\end{array}$ \\
\hline Gover (2003) & $\begin{array}{c}N=509 \text { chicos } \\
\text { Edad media }=16 \\
\text { Detenidos y cumpliendo condena }\end{array}$ & EE.UU. & $\begin{array}{c}\text { Maltrato infantil (abuso sexual, físico, } \\
\text { negligencia...) }\end{array}$ & Depresión & $\begin{array}{l}\text { Los jóvenes encarcelados con historia de maltrato infantil } \\
\text { tuvieron niveles más altos de ansiedad y depresión, manteniendo } \\
\text { constantes otros factores relacionados (individuales e } \\
\text { institucionales). }\end{array}$ \\
\hline Gover (2004) & $\begin{array}{c}N=583(377 \text { chicos y } 206 \text { chicas }) \\
\text { Edad media }=15,7 \\
\text { Detenidos } / \text { as }\end{array}$ & EE.UU. & Abuso sexual & Depresión & $\begin{array}{l}\text { Los jóvenes sexualmente abusados en la infancia tenían más } \\
\text { tasas de depresión, siendo más altas para las mujeres. }\end{array}$ \\
\hline Hill et al. (2014) & $\begin{array}{c}N=30 \text { chicas } \\
\text { Edad media }=16,41 \\
\text { Detenidas y cumpliendo condena }\end{array}$ & $\begin{array}{l}\text { Reino Unido } \\
\text { (Inglaterra) }\end{array}$ & Abuso sexual y exposición a violencia & TEPT & $\begin{array}{l}\text { En muchas de las niñas se encontró evidencia de TEPT, los } \\
\text { datos recabados indican abuso sexual en el pasado y exposición } \\
\text { a violencia como algo común en las pacientes. }\end{array}$ \\
\hline Hoeve et al. (2015) & $\begin{array}{l}N=422 \text { chicos } \\
\text { Edad media }=16,7 \\
\quad \text { Detenidos }\end{array}$ & Países Bajos & Abuso sexual y negligencia & $\begin{array}{l}\text { Síntomas depresivos/ } \\
\text { ansiosos }\end{array}$ & $\begin{array}{l}\text { Se encontró relación entre maltrato infantil y problemas de salud } \\
\text { mental, siendo mayores las correlaciones entre negligencia } \\
\text { emocional e irritabilidad, o depresión/ansiosa. El abuso sexual } \\
\text { sólo se asoció significativamente a irritabilidad y problemas } \\
\text { depresivos/ansiosos. }\end{array}$ \\
\hline
\end{tabular}


Morales-Toro, V., Guillén-Riquelme, A. y Quevedo-Blasco, R.

\begin{tabular}{|c|c|c|c|c|c|}
\hline $\begin{array}{l}\text { Hunter, Figuered, } \\
\text { Malamut y Becker } \\
\quad(2003)\end{array}$ & $\begin{array}{l}\quad N=182 \text { chicos } \\
\text { Edad media }=15,9 \\
\quad \text { Detenidos }\end{array}$ & EE.UU. & Abuso físico & Depresión & $\begin{array}{l}\text { Se encontró relación entre abuso físico infantil y exposición a } \\
\text { violencia en contra de mujeres y mayores niveles de ansiedad y } \\
\text { depresión comórbidas. }\end{array}$ \\
\hline $\begin{array}{l}\text { Jencks y Leibowitz } \\
\text { (2018) }\end{array}$ & $\begin{array}{l}N=420 \text { chicos } \\
\text { Edad media }=16,7 \\
\quad \text { Detenidos }\end{array}$ & $\begin{array}{l}\text { EE.UU. (Medio } \\
\text { Oeste y Este) }\end{array}$ & $\begin{array}{l}\text { Abuso físico, emocional o sexual, y } \\
\text { negligencia física y emocional }\end{array}$ & Depresión & $\begin{array}{l}\text { Se encontró asociación entre los diferentes tipos de traumas, } \\
\text { pero el mayor predictor fue el abuso emocional. La cantidad de } \\
\text { trauma no fue predictor tan fuerte de la depresión como el abuso } \\
\text { emocional. }\end{array}$ \\
\hline $\begin{array}{c}\text { Kerig y } \\
\text { Modrowski (2018) }\end{array}$ & $\begin{array}{c}N=782(579 \text { chicos y } 203 \text { chicas }) \\
\text { Edad media }=16,07 \\
\text { Detenidos } / \text { as }\end{array}$ & EE.UU. (Oeste) & Polivictimización & $\begin{array}{l}\text { Trastorno límite de } \\
\text { personalidad }\end{array}$ & $\begin{array}{l}\text { Las niñas mostraron niveles más altos de polivictimización, } \\
\text { personalidad límite y disociación, que los niños. Dentro de los } \\
\text { efectos indirectos la polivictimización y personalidad límite se } \\
\text { vincularon a través de la disociación. }\end{array}$ \\
\hline King et al. (2011) & $\begin{array}{c}N=1.735(1.095 \text { chicos y } 640 \\
\text { chicas }) \\
\text { Edad media }=14,8 \\
\text { Detenidos } / \text { as }\end{array}$ & $\begin{array}{l}\text { EE.UU. } \\
\text { (Condado de } \\
\text { Cook) }\end{array}$ & Abuso físico y sexual & Trastornos afectivos & $\begin{array}{l}\text { Las mujeres maltratadas con fuerza tuvieron índices más altos de } \\
\text { ansiedad y trastornos afectivos, en cambio en los hombres se } \\
\text { asoció a TDAH y trastorno por uso de sustancias. }\end{array}$ \\
\hline $\begin{array}{l}\text { Lansing, Plante, Beck } \\
\text { y Ellenberg (2018) }\end{array}$ & $\begin{array}{l}N=107 \text { (51 chicos y } 56 \text { chicas }) \\
\text { Edad media }=17,16 \text { y } 16,57 \\
\text { respectivamente } \\
\text { Detenidos/as }\end{array}$ & EE.UU. & Abuso emocional, físico y sexual & $\begin{array}{c}\text { Síntomas } \\
\text { internalización (ansioso/ } \\
\text { depresivo y abandono/ } \\
\text { deprimido) }\end{array}$ & $\begin{array}{l}\text { Los síndromes de internacionalización se asociaron al abuso } \\
\text { emocional y físico, en las chicas además al abuso sexual. }\end{array}$ \\
\hline Leduc (2002) & $\begin{array}{l}N=70(50 \text { chicos y } 20 \text { chicas }) \\
\text { Edad media }=15,53 \\
\text { Detenidos } / \text { as }\end{array}$ & $\begin{array}{l}\text { EE.UU. } \\
\text { (Condado de } \\
\text { Fresno) }\end{array}$ & Trauma & TEPT & $\begin{array}{l}\text { Participantes con TEPT habían experimentado más traumas que } \\
\text { los no TEPT. El número de traumas notificados se correlacionó } \\
\text { con el número de síntomas de TEPT y la gravedad de los } \\
\text { síntomas. Los traumas más asociados fueron: tortura, agresión } \\
\text { sexual por persona conocida, por un desconocido, que sea a edad } \\
\text { temprana y luego otros eventos donde el daño directo es a otras } \\
\text { personas. }\end{array}$ \\
\hline Lemos y Faísca (2015) & $\begin{array}{c}N=50 \text { chicos } \\
\text { Edad media }=15,82 \\
\text { Detenidos y cumpliendo condena }\end{array}$ & Portugal & $\begin{array}{c}\text { Abuso infantil, maltrato, negligencia, } \\
\text { institucionalización, adicción } \\
\text { familiar... }\end{array}$ & TEPT y depresión & $\begin{array}{l}\text { Los adolescentes detenidos reportaron problemas de } \\
\text { internalización (TEPT, depresión, problemas interpersonales y } \\
\text { ansiedad) significativamente más altos que aquellos que asisten } \\
\text { a equipos basados en la comunidad, lo que es indicativo de } \\
\text { mayor exposición a factores estresantes altamente negativos y } \\
\text { continuos, como el abuso de los padres y relaciones familiares } \\
\text { conflictivas. }\end{array}$ \\
\hline $\begin{array}{l}\text { Lindberg, Oshukov, } \\
\text { Miettunen y Kaltiala- } \\
\text { Heino (2016) }\end{array}$ & $\begin{array}{c}N=266(241 \text { chicos y } 25 \text { chicas }) \\
\text { Edad media }=16,3 \\
\text { Detenidos } / \text { as }\end{array}$ & Finlandia & Abuso sexual y físico & Rasgos psicopáticos & $\begin{array}{l}\text { Dos tercios de ambos sexos exhibieron rasgos psicopáticos } \\
\text { elevados y un tercio altos rasgos de psicopatía, pudiendo estar } \\
\text { relacionado con historias de abuso y trauma. Las niñas habían } \\
\text { sido más a menudo objeto de abuso sexual y objeto de violencia } \\
\text { física en la infancia. }\end{array}$ \\
\hline $\begin{array}{l}\text { Matsuura, } \\
\text { Hashimoto } \\
\text { y Toichi } \\
\text { (2013) }\end{array}$ & $\begin{array}{c}N=81 \text { clínicos }(\operatorname{chicos} / \text { as }) ; 285 \\
\text { control }(\text { chicos } / \text { as }) \\
\text { Edad media }=17 \\
\text { Detenidos } / \text { as }\end{array}$ & Japón & $\begin{array}{c}\text { Diferentes experiencias traumáticas en } \\
\text { la infancia }\end{array}$ & Depresión & $\begin{array}{l}\text { Las puntuaciones de agresión, ETI y depresión, correlacionaron } \\
\text { positivamente entre sí. Los trastornos emocionales cuando son } \\
\text { agravados por las ETI, interactúan estrechamente entre sí y } \\
\text { están directamente relacionados con la baja autoestima. }\end{array}$ \\
\hline
\end{tabular}




\begin{tabular}{|c|c|c|c|c|c|}
\hline $\begin{array}{c}\text { Mcpeek } \\
(1997)\end{array}$ & $\begin{array}{l}N=112(74 \text { chicos y } 38 \text { chicas }) \\
\text { Edad media }=15,97 \text { y } 16 \\
\text { respectivamente } \\
\text { Cumpliendo condena }\end{array}$ & EE.UU. & $\begin{array}{c}\text { Diferentes experiencias traumáticas en } \\
\text { la infancia }\end{array}$ & TEPT & $\begin{array}{l}\text { En los hombres el trauma fue un predictor del TEPT, en el } 85 \% \\
\text { de las veces. La experiencia de abuso sexual y trauma predijeron } \\
\text { correctamente el resultado de un diagnóstico de TEPT con } \\
73,7 \% \text { de precisión, en el caso de las mujeres ya que los } \\
\text { hombres relataron no haber sufrido abuso sexual. }\end{array}$ \\
\hline Moore (2003) & $\begin{array}{c}N=57 \text { chicos } / \text { as y } 38 \text { grupo control } \\
\text { Edad media }=14,46 \\
\text { Cumpliendo condena }\end{array}$ & EE.UU. & Abuso sexual & Depresión & $\begin{array}{l}\text { Los jóvenes sexualmente victimizados tenían mayores tasas de } \\
\text { depresión que los no sexualmente victimizados, aunque hay que } \\
\text { tener en cuenta las diferencias entre depresión autoinformada y } \\
\text { la de los informes de cuidadores. }\end{array}$ \\
\hline $\begin{array}{l}\text { Moore, Gaskin y Indig } \\
\text { (2013) }\end{array}$ & $\begin{array}{c}N=291(253 \text { chicos y } 38 \text { chicas }) \\
\text { Edad media }=17 \\
\text { Detenidos } / \text { as }\end{array}$ & Australia & $\begin{array}{l}\text { Maltrato infantil: abuso (sexual, físico, } \\
\text { emocional) y negligencia (emocional y } \\
\text { física) }\end{array}$ & TEPT & $\begin{array}{l}\text { El antecedente de trauma infantil fue el principal predictor de } \\
\text { TEPT. El abuso emocional fue el más frecuente y el sexual el } \\
\text { menos frecuente. Las mujeres tenían más probabilidad de } \\
\text { reportar trauma infantil y más tasas de TEPT. }\end{array}$ \\
\hline $\begin{array}{l}\text { Morais, Alexander, } \\
\text { Fix y Burkhart (2018) }\end{array}$ & $\begin{array}{c}N=498 \text { chicos } \\
\text { Edad media }=15,93 \\
\quad \text { Detenidos }\end{array}$ & $\begin{array}{l}\text { EE.UU. (Estado } \\
\text { del sudeste) }\end{array}$ & Abuso sexual infantil & TEPT y depresión & $\begin{array}{l}\text { Los chicos con historia de abuso sexual eran más propensos a } \\
\text { tener depresión mayor y TEPT, esta relación no se encontró para } \\
\text { la ansiedad. }\end{array}$ \\
\hline $\begin{array}{l}\text { Nowakowski-Sims y } \\
\text { Rowe (2017) }\end{array}$ & $\begin{array}{c}N=80(42 \text { chicos y } 38 \text { chicas }) \\
\text { Edad media }=15 \\
\text { Cumpliendo condena }\end{array}$ & EE.UU. & $\begin{array}{l}\text { Diferentes experiencias traumáticas en } \\
\text { la infancia }\end{array}$ & Depresión & $\begin{array}{l}\text { Se encontró que la experiencia previa con violencia en el hogar e } \\
\text { interrupciones con el apego a la madre, son variables } \\
\text { fundamentales para la predicción de la depresión. }\end{array}$ \\
\hline $\begin{array}{l}\text { Perez, Jennings y } \\
\text { Baglivio (2018) }\end{array}$ & $\begin{array}{c}N=64.329(50,389 \text { chicos y } 13,940 \\
\text { chicas }) \\
\text { Edad media }=17 \\
\text { Cumpliendo condena }\end{array}$ & $\begin{array}{l}\text { EE.UU. } \\
\text { (Florida) }\end{array}$ & $\begin{array}{c}\text { Diferentes experiencias traumáticas en } \\
\text { la infancia }\end{array}$ & $\begin{array}{l}\text { Rasgos de personalidad } \\
\text { impulsiva }\end{array}$ & $\begin{array}{l}\text { Una mayor puntuación en experiencias traumáticas en la } \\
\text { infancia, se asoció a mayor impulsividad. Destacar que la } \\
\text { impulsividad fue mayor en los chicos. }\end{array}$ \\
\hline $\begin{array}{l}\text { Roe-Sepowitz y } \\
\text { Krysik (2008) }\end{array}$ & $\begin{array}{c}\quad N=118 \text { chicas } \\
\text { Edad media }=13,9 \\
\text { Cumpliendo condena }\end{array}$ & $\begin{array}{l}\text { EE.UU. } \\
\text { (Florida) }\end{array}$ & $\begin{array}{l}\text { Abuso físico y sexual, negligencia y } \\
\text { múltiples abusos }\end{array}$ & Depresión & $\begin{array}{l}\text { Los delincuentes sexuales juveniles con maltrato infantil tenían } \\
\text { más probabilidades de tener un diagnóstico actual de salud } \\
\text { mental y experimentar niveles clínicos de depresión-ansiedad. }\end{array}$ \\
\hline $\begin{array}{c}\text { Ručević y Ajduković } \\
\text { (2016) }\end{array}$ & $\begin{array}{c}N=67 \text { grupo clínico }(47 \text { chicos y } 20 \\
\text { chicas }) ; 78 \text { grupo control } \\
\text { Edad media }=16,30 \\
\text { Detenidos } / \text { as }\end{array}$ & Croacia & $\begin{array}{l}\text { Diferentes experiencias traumáticas en } \\
\text { la infancia y victimización física y } \\
\text { sexual }\end{array}$ & Rasgos psicopáticos & $\begin{array}{l}\text { Las personas detenidas con rasgos psicopáticos informaron } \\
\text { mayores tasas de experiencias traumáticas en la infancia y } \\
\text { victimización física, sin embargo, no difirieron en victimización } \\
\text { sexual. }\end{array}$ \\
\hline $\begin{array}{l}\text { Ruffolo, Sarri y } \\
\text { Goodkind (2004) }\end{array}$ & $\begin{array}{c}N=159 \text { chicas } \\
\text { Edad media }=15,8 \\
\text { Detenidas y cumpliendo condena }\end{array}$ & $\begin{array}{c}\text { EE.UU. } \\
\text { (Michigan) }\end{array}$ & $\begin{array}{l}\text { Abuso sexual y otras experiencias } \\
\text { traumáticas en la infancia }\end{array}$ & Depresión & $\begin{array}{l}\text { En esta investigación se encontró una asociación positiva entre } \\
\text { depresión y diferentes experiencias traumáticas y haber sufrido } \\
\text { abuso sexual. }\end{array}$ \\
\hline $\begin{array}{l}\text { Silva, Graña y } \\
\text { González-Cieza } \\
\text { (2014) }\end{array}$ & $\begin{array}{l}N=104(94 \text { chicos y } 10 \text { chicas }) \\
\text { Edad media }=16,5 \\
\text { Detenidos } / \text { as }\end{array}$ & España & Abuso (físico y emocional) & $\begin{array}{l}\text { Internalización } \\
\text { (depresión) }\end{array}$ & $\begin{array}{l}\text { Se encontró relación entre abuso físico y problemas de } \\
\text { internalización y externalización. El abuso emocional se asoció a } \\
\text { problemas de externalización, incluso incluyendo insensibilidad } \\
\text { emocional, como moderador. }\end{array}$ \\
\hline
\end{tabular}




\begin{tabular}{|c|c|c|c|c|c|}
\hline $\begin{array}{l}\text { Taylor, James, Reeves } \\
\text { y Kistner (2009) }\end{array}$ & $\begin{array}{c}N=239 \text { grupo clínico chicos; } \\
\text { 1.197grupo control chicos } \\
\text { Edad media }=16,3 \\
\text { Detenidos }\end{array}$ & $\begin{array}{l}\text { EE.UU. } \\
\text { (Florida) }\end{array}$ & Abuso sexual y físico & TPL & $\begin{array}{l}\text { Una proporción mayor de adolescentes con rasgos de } \\
\text { personalidad límite, habían sido sexual y físicamente más } \\
\text { abusados que el grupo control (no TPL). }\end{array}$ \\
\hline $\begin{array}{c}\text { Tossone, Wheeler, } \\
\text { Butcher y Kretschmar } \\
\text { (2018) }\end{array}$ & $\begin{array}{c}N=1.307 \text { chicas } \\
\text { Edad media }=15,28 \\
\text { Cumpliendo condena }\end{array}$ & EE.UU. (Ohio) & Abuso sexual & Depresión & $\begin{array}{l}\text { Las mujeres sexualmente abusadas, tenían mayores puntuaciones } \\
\text { de traumas psicológicos, especialmente depresión y otras } \\
\text { consecuencias no específicas para esta revisión. }\end{array}$ \\
\hline Vahl et al. (2016) & $\begin{array}{c}N=439 \text { chicos } \\
\text { Edad media }=16,5 \\
\text { Detenidos }\end{array}$ & Países Bajos & $\begin{array}{l}\text { Abuso (físico, sexual y emocional), } \\
\text { abandono físico }\end{array}$ & Psicopatía & $\begin{array}{l}\text { Para aquellos con bajos rasgos psicopáticos, el maltrato tuvo } \\
\text { más impacto que para los que tenían altos niveles de rasgos } \\
\text { psicopáticos. Hubo una relación positiva entre tipos de maltrato } \\
\text { y rasgos psicopáticos, siendo moderada la relación con el abuso } \\
\text { emocional y débil con el resto de abuso. }\end{array}$ \\
\hline $\begin{array}{l}\text { Vahl, van Damme, } \\
\text { Doreleijers, Vermeireny } \\
\text { Colins (2016) }\end{array}$ & $\begin{array}{c}N=156 \text { chicos } ; 185 \text { chicas } \\
\text { Edad media }=15,9 \\
\text { Detenidos } / \text { as }\end{array}$ & Bélgica & $\begin{array}{c}\text { Abuso (físico, sexual y emocional), } \\
\text { negligencia (física y emocional) }\end{array}$ & $\begin{array}{l}\text { Internalización } \\
\text { (retraído/ deprimido, } \\
\text { ansioso/ depresivo) }\end{array}$ & $\begin{array}{l}\text { Se encontraron fuertes relaciones entre abuso emocional y } \\
\text { problemas de internalización, en ambos géneros. En los chicos } \\
\text { se asoció la internalización débilmente a negligencia emocional } \\
\text { y moderadamente al abuso físico. En las niñas se obtuvo } \\
\text { relación moderada en abuso sexual y problemas de } \\
\text { internalización. }\end{array}$ \\
\hline $\begin{array}{l}\text { Wanklyn, Day, Hart y } \\
\text { Girard (2012) }\end{array}$ & $\begin{array}{c}N=110(67 \text { chicos y } 43 \text { chicas }) \\
\text { Edad media }=16,78 \\
\text { Cumpliendo condena }\end{array}$ & Canadá & Maltrato infantil & Depresión & $\begin{array}{l}\text { Los resultados del estudio indicaron que el maltrato acumulativo } \\
\text { durante la infancia, se asoció a mayores niveles de desesperanza, } \\
\text { lo cual se relacionó a su vez con la gravedad de la depresión. }\end{array}$ \\
\hline
\end{tabular}

Nota. $\mathrm{N}=$ muestra; $\mathrm{NM}=$ no trauma o trauma leve; $\mathrm{EP}=$ trauma emocional y físico; $\mathrm{EPS}=$ trauma emocional, físico y sexual; TEPT= trastorno de estrés postraumático; ETI= experiencias traumáticas en la infancia; $\mathrm{JSO}=$ jóvenes que han ofendido sexualmente; $\mathrm{No} \mathrm{JSO}=$ jóvenes que no han ofendido sexualmente pero sí han realizado otros delitos; TDAH= trastorno por déficit de atención e hiperactividad; TPL= trastorno límite de la personalidad; EE.UU.: Estados Unidos de América.

* Los estudios han sido ordenados alfabéticamente por el apellido del primer autor.

Tabla 1. Cuadro resumen de los datos característicos de los estudios analizados 


\subsection{Análisis de los estudios}

Es importante conocer que la proporción de chicos y chicas que se han evaluado en sus respectivos estudios, no son equiparables. El 75,55\% de los estudios han usado una muestra de chicos frente al $23,30 \%$ que han usado una muestra de chicas, teniendo en cuenta que un $1,15 \%$ de los documentos no han hecho diferencias por razón de sexo.

Dentro de los países que se han evaluado (se ha tomado una muestra de estudios a nivel mundial), se ha detectado que el mayor porcentaje de estudios pertenecen a EE.UU. con un 48,84\%, al que le siguen Países Bajos y Australia 6,98\% cada uno, Japón y Canadá 4,65\%, y países como Austria, Suiza, Chile, Malasia, Reino Unido, Portugal, Finlandia, Croacia, Bélgica y España, los cuales tienen el menor porcentaje de todos los países, obteniendo así un 2,33\% de los estudios en cada país.

A continuación, se va a hacer mención a los trastornos que se suelen dar en mayor porcentaje para la población elegida, teniendo en cuenta que, en un mismo estudio, en ocasiones se han evaluado y por tanto se han tenido en cuenta más de un trastorno y tipo de abuso. De los 43 estudios que contienen información sobre trastornos vinculados a experiencias traumáticas en la infancia, el 69,04\% (29) muestra alta tasa de trastornos del humor (afectivos), siendo dentro de éstos la depresión y problemas depresivos ansiosos 93,1\% (27) los más frecuentes, seguida de otros trastornos afectivos no especificados en el estudio 6,89\% (2). El trastorno de estrés postraumático fue el siguiente en mayor porcentaje, obteniendo así un 27,9\% del total de estudios (12). Por último, se encontraron los trastornos de personalidad con un $18,6 \%$ (8), dentro de ellos, la psicopatía o rasgos psicopáticos se dio en mayor proporción $62,5 \%$ (5), seguida del trastorno límite de la personalidad 28,57\% (2) y el trastorno de personalidad impulsivo $14,28 \%$ (1).

Dentro del maltrato o abuso recibido en la infancia con los que se encontró relación, se ha obtenido que el abuso sexual y el sufrir diferentes experiencias traumáticas en la infancia fueron los más prevalentes, con un 55,81\% (24) de los estudios, cada uno. A esto le seguiría el abuso físico 44,19\% (19) y por último el abuso emocional 30,23\% (13).

Asimismo se hace preciso remarcar que dentro de los trastornos del humor (afectivos), se dio un mayor porcentaje de abuso sexual 55,17\% (16), experiencias traumáticas de diversa índole 44,82\% (13), abuso físico 41,38\% (12) y en último lugar el abuso emocional 31,03\% (9). En el caso del TEPT, las diferentes experiencias traumáticas también fueron más frecuentes 75\% (9), seguidas del abuso sexual $50 \%(6)$, abuso físico 25\% (3) y abuso emocional 16,66\% (2). En último lugar en los trastornos de personalidad se encontró mayor relación con las diferentes experiencias traumáticas en la infancia y abuso físico, 62,5\% (5) cada uno, y abuso emocional y sexual con un $37,5 \%$ (3), en ambos igual proporción.

\section{DISCUSIÓN}

El objetivo de esta revisión ha sido analizar la posible asociación entre experiencias traumáticas en la infancia y el desarrollo de determinados trastornos en delincuentes juveniles, a nivel mundial. En total se han analizado 43 documentos. Es necesario tener 
en cuenta que no se ha incluido otra revisión afín (Foy, Ritchie y Conway, 2012), por no cumplir con los criterios de inclusión/exclusión necesarios (ya que incluyen diferentes tipos de traumas que no acontecieron únicamente en la infancia; además no diferencian qué tipo de trauma es el más vinculado al trastorno, si el infantil o el de otra etapa de la vida). Los resultados respecto al trastorno indican que el afectivo es el más frecuente, asimismo en un estudio de 1.829 delincuentes, se encontró que entre los diagnósticos frecuentes se encontraban los trastornos afectivos (Teplin, Abram, McClelland, Dulcan y Mericle, 2002). Dentro de éstos el más común es el trastorno depresivo (Adams et al., 2013; Berzenski y Yates, 2011). Siguiendo con los trastornos más frecuentes, tenemos el TEPT (Abram et al., 2013; Breslau et al., 2014; Foy et al., 2012). En referencia a los trastornos de personalidad y sobre todo haciendo mención a la psicopatía o rasgos psicopáticos como su mayor expresión, se ha hallado otro estudio que destaca a la psicopatía o rasgos psicopáticos (Tuvblad, Bezdjian, Raine y Baker, 2013). Destacar que estos trastornos también son objeto de estudio en adolescentes no delincuentes (Barrera-Valencia, Calderón-Delgado, Trejos-Castillo y O’Boylec, 2017; Ho, Wai Tong Dai, Mak y Wing Kei Liu, 2018).

Atendiendo a los tipos de abusos en la infancia, se encuentra que el más prevalente fue el abuso sexual y las diferentes experiencias traumáticas en la infancia (Ford, Grasso, Hawke y Chapman, 2013; Putnam, 2003; Veneziano, Veneziano y Legrand, 2000). En contraposición, otros autores han resaltado con más frecuencia al abuso físico y emocional (Vallejos et al., 2014; Wolff y Shi, 2012). En este sentido, también es importante tener en cuenta cuales son los predictores de las agresiones sexuales en los adolescentes (Moyano, Monge y Sierra, 2017).

Respecto a la relación trastornos afectivos y abuso sexual, hay resultados similares en el estudio de Coleman (2005), un estudio que se realizó a 74 adolescentes encarcelados y en el cual hubo una fuerte asociación del abuso sexual sobre el suicidio y problemas de internalización. Además, Hyun, Friedman y Dunner (2000) relacionaron el trastorno bipolar con experiencia de abuso sexual en la infancia. En contraposición Fernández-Martínez et al. (2018), asociaron el maltrato físico y trastornos afectivos, concretamente la depresión.

Como en este estudio, el TEPT se asoció a diferentes experiencias traumáticas en la infancia (Frewen, Zhu y Lanius, 2019). En el estudio llevado a cabo por Stimmel et al. (2014) probaron que los delincuentes juveniles expuestos a múltiples tipos de trauma o una exposición que implica violencia, reportaron mayores niveles de sintomatología de TEPT. Respecto a la asociación trastornos de personalidad y experiencias traumáticas, los siguientes estudios encontraron datos similares; en primer lugar, señalar el estudio de Forouzan y Nicholls (2015), donde se asociaron diversas formas de victimización a altas puntuaciones en rasgos psicopáticos; y donde además de esto, se ha encontrado una asociación entre la gravedad del maltrato infantil y la gravedad de algunos trastornos de personalidad en la edad adulta (Dargis, Newman y Koenigs 2016).

En este estudio es necesario tener en cuenta una serie de limitaciones. En primer lugar que la mayoría de estudios incluidos eran retrospectivos, con lo cual no se podían realizar asociaciones de causalidad entre los resultados. Otro aspecto es que los delincuentes en muchos de los estudios habían cometido a su vez delitos sexuales y como se puede apreciar en un trabajo de Craissati, McClurg y Browne (2002), puede que haya mayor prevalencia de antecedentes de abuso sexual en agresores sexuales. La mayoría de los datos son recabados de EE.UU. y en muchos de los estudios no 
mencionaban el manual que habían usado para el diagnóstico de la muestra. Por tanto, son resultados que deben asumirse teniendo cierta precaución. Para futuros estudios puede ser interesante tener en cuenta otras variables asociadas a la agresión en los adolescentes (ver, por ejemplo, Álvarez-García, Núñez, García y Barreiro-Collazo, 2018; Cutrín, Gómez-Fraguela, Maneiro y Sobral, 2017; Jiménez y Estévez, 2017; López-Pérez, 2017). Además de esto, se encuentran puntos fuertes como diferentes trastornos y tipos de abusos que se suelen asociar a la población delincuente en un solo estudio, así como conocer los más frecuentes y las asociaciones entre sí. Por otra parte, las bases de datos utilizadas, el rango de años, el hecho de que se haya hecho a nivel mundial y se haya pasado una escala de calidad a cada documento y un análisis de fiabilidad, permite obtener una información más completa y de calidad.

Para concluir, en los resultados obtenidos se puede ver una mayor tasa de hombres estudiados (respecto a mujeres), y que los trastornos del humor (afectivos) son los que se suelen dar en mayor proporción en la población delincuente analizada. Dentro de los abusos, el abuso sexual en la infancia o diferentes experiencias traumáticas fueron los hechos más prevalentes, con lo cual estos son también los más asociados a los trastornos afectivos, trastornos de personalidad y TEPT.

\section{BIBLIOGRAFÍA}

Las referencias marcadas con un asterisco (*) indican que han sido incluidas en la revisión sistemática.

Abram, K.M., Teplin, L.A., King, D.C., Longworth, S.L., Emanuel, K.M., Romero, E.G. y Olson, N.D. (2013). PTSD, trauma, and comorbid psychiatric disorders in detained youth. Washington, D.C.: Office of Juvenile Justice and Delinquency Prevention. Recuperado de: https://www.ojjdp.gov/pubs/239603.pdf

Adams, Z.W., McCart, M.R., Zajac, K., Danielson, C.K., Sawyer, G.K., Saunders, B.E. y Kilpatrick, D.G. (2013). Psychiatric problems and trauma exposure in nondetained delinquent and nondelinquent adolescents. Journal of Clinical Child \& Adolescent Psychology, 42 (3), 323-331. http://dx.doi.org/10.1080/15374416.2012.749786

*Aebi, M., Linhart, S., Thun-Hohenstein, L., Bessler, C., Steinhausen, H.C. y Plattner, B. (2015). Detained male adolescent offender's emotional, physical and sexual maltreatment profiles and their associations to psychiatric disorders and criminal behaviors. Journal of Abnormal Child Psychology, 43 (5), 999-1009. http://dx.doi.org/10.1007/s10802-014-9961-y

Alarcón, P.A., Pérez-Luco, R.X., Wenger, L.S., Salvo, S.I. y Chesta, S.A. (2018). Personalidad y gravedad delictiva en adolescentes con conducta antisocial persistente. Revista Iberoamericana de Psicología y Salud, 9 (1), 58-74. http://dx.doi.org/10.23923/j.rips.2018.01.015

Álvarez-García, D., Núñez, J.C., García, T. y Barreiro-Collazo, A. (2018). Individual, family, and community predictors of cyber-aggression among adolescents. European Journal of Psychology Applied to Legal Context, 10 (2), 79-88. http://dx.doi.org/10.5093/ejpalc2018a8

*Ariga, M., Uehara, T., Takeuchi, K., Ishige, Y., Nakano, R. y Mikuni, M. (2008). Trauma exposure and posttraumatic stress disorder in delinquent female adolescents. Journal of Child Psychology and Psychiatry and Allied Disciplines, 49 (1), 79-87.

http://dx.doi.org/10.1111/j.1469-7610.2007.01817.x 
Baglivio, M., Epps, N., Swartz, K., Huq, M.S., Sheer, A. y Hardt, N.S. (2014). The prevalence of Adverse Childhood Experiences (ACE) in the lives of juvenile offenders. Journal of Juvenile Justice, 3 (2), 1-23. Recuperado de:

https://pdfs.semanticscholar.org/9466/b7ff1b7dd08ecb668a5836f89ed03d3638d5.pdf

Barrera-Valencia, M., Calderón-Delgado, L., Trejos-Castillo, E. y O’Boylec, M. (2017). Cognitive profiles of Post-Traumatic Stress Disorder and depression in children and adolescents. International Journal of Clinical and Health Psychology, 17 (3), 242-250. http://dx.doi.org/10.1016/j.ijchp.2017.05.001

Becerra-García, J.A. y García-León, A. (2012). Trastorno mental en los agresores sexuales: estudio descriptivo de una muestra española. Revista Española de Medicina Legal, 39 (1), 3-6. http://dx.doi.org/10.1016/j.reml.2012.10.007

Berzenski, S.R. y Yates, T.M. (2011). Classes and consequences of multiple maltreatment: A person-centered analysis. Child Maltreatment, 16 (4), 250-261. http://dx.doi.org/10.1177/1077559511428353

*Bielas, H., Barra, S., Skrivanek, C., Aebi, M., Steinhausen, H.C., Bessler, C. y Plattner, B. (2016). The associations of cumulative adverse childhood experiences and irritability with mental disorders in detained male adolescent offenders. Child and Adolescent Psychiatry and Mental Health, 10 (1), 1-10. http://dx.doi.org/10.1186/s13034-016-0122-7

*Boonmann, C., Grisso, T., Guy, L.S., Colins, O.F., Mulder, E.A., Vahl, P.,...Vermeiren, R. (2016). Childhood traumatic experiences and mental health problems in sexually offending and non-sexually offending juveniles. Child and Adolescent Psychiatry and Mental Health, 10 (1), 1-8. http://dx.doi.org/10.1186/s13034-016-0127-2

Breslau, N., Koenen, K.C., Luo, Z., Agnew-Blais, J., Swanson, S., Houts, R. M.,...Moffitt, T.E. (2014). Childhood maltreatment, juvenile disorders and adult post-traumatic stress disorder: A prospective investigation. Psychological Medicine, 44 (9), 1937-1945. http://dx.doi.org/10.1017/S0033291713002651

Caravaca Sánchez, F., Ignatyev, Y. y Mundt, A.P. (2018). Associations between childhood abuse, mental health problems, and suicide risk among male prison populations in Spain. Criminal Behaviour and Mental Health, 29 (1), 18-30. http://dx.doi.org/10.1002/cbm.2099

Cerezo, M.A. y Vera, P. (2004). Antecedentes de maltrato infantil en la conducta antisocial y delictiva autoinformada: Un estudio con menores infractores. Bienestar y Protección Infantil, 3 (2), 41-60. Recuperado de: https://www.bienestaryproteccioninfantil.es/imagenes/tablaContenidos03SubSec/Revista_V ol3_Num2_Completo.pdf

Clements-Nolle, K., Larson, S., Buttar, A. y Dermid-Gray, L. (2017). Childhood maltreatment and unprotected sex among female juvenile offenders: Evidence of mediation by substance abuse and psychological distress. Women's Health Issues, 27 (2), 188-195. http://dx.doi.org/10.1016/j.whi.2016.12.004

Coleman, D. (2005). Trauma and incarcerated youth. Journal of Evidence-Based Social Work, 2 (3-4), 113-124. http://dx.doi.org/10.1300/J394v02n03_08

*Coleman, D. y Stewart, L.M. (2010). Prevalence and impact of childhood maltreatment in incarcerated youth. American Journal of Orthopsychiatry, 80 (3), 343-349. http://dx.doi.org/10.1111/j.1939-0025.2010.01038.x

Craissati, J., McClurg, G. y Browne, K. (2002). Characteristics of perpetrators of child sexual abuse who have been sexually victimized as children. Sexual Abuse: A Journal of Research and Treatment, 14 (3), 225-239. http://dx.doi.org/10.1177/107906320201400303

Cutrín, O., Gómez-Fraguela, J.A., Maneiro, L. y Sobral, J. (2017). Effects of parenting practices through deviant peers on nonviolent and violent antisocial behaviours in middle- and lateadolescence. European Journal of Psychology Applied to Legal Context, 9 (2), 75-82. http://dx.doi.org/10.1016/j.ejpal.2017.02.001 
Dargis, M., Newman, J. y Koenigs, M. (2016). Clarifying the link between childhood abuse history and psychopathic traits in adult criminal offenders. Personality Disorders: Theory, Research, and Treatment, 7 (3), 221-228. http://dx.doi.org/10.1037/per0000147

*Daversa, M.T. y Knight, R.A. (2007). A structural examination of the predictors of sexual coercion against children in adolescent sexual offenders. Criminal Justice and Behavior, 34 (10), 1.313-1.333. http://dx.doi.org/10.1177/0093854807302411

*Day, D.M., Hart, T.A., Wanklyn, S.G., McCay, E., Macpherson, A. y Burnier, N. (2013). Potential mediators between child abuse and both violence and victimization in juvenile offenders. Psychological Services, 10 (1), 1-11. http://dx.doi.org/10.1037/a0028057

Dias, A., Sales, L., Mooren, T., Mota-Cardoso, R. y Kleber, R. (2017). Child maltreatment, revictimization and post-traumatic stress disorder among adults in a community sample. International Journal of Clinical and Health Psychology, 17 (2), 97-106. http://dx.doi.org/10.1016/j.ijchp.2017.03.003

*Dierkhising, C.B., Ko, S.J., Woods-Jaeger, B., Briggs, E.C., Lee, R. y Pynoos, R.S. (2013). Trauma histories among justice-involved youth: Findings from the National Child Traumatic Stress Network. European Journal of Psychotraumatology, 4 (1), 20.274. http://dx.doi.org/10.3402/ejpt.v4i0.20274

*Dixon, A., Howie, P. y Starling, J. (2004). Psychopathology in female juvenile offenders. Journal of Child Psychology and Psychiatry and Allied Disciplines, 45 (6), 1150-1158. http://dx.doi.org/10.1111/j.1469-7610.2004.00307.x

*Dixon, A., Howie, P. y Starling, J. (2005). Trauma exposure, posttraumatic stress, and psychiatric comorbidity in female juvenile offenders. Journal of the American Academy of Child and Adolescent Psychiatry, 44 (8), 798-806. http://dx.doi.org/10.1097/01.chi.0000164590.48318.9c

*Farina, A.S.J., Holzer, K.J., DeLisi, M. y Vaughn, M.G. (2018). Childhood trauma and psychopathic features among juvenile offenders. International Journal of Offender Therapy and Comparative Criminology, 62 (14), 4.359-4.380. http://dx.doi.org/10.1177/0306624X18766491

Fariña, F., Arce, R. y Novo, M. (2002). Heurístico de anclaje en las decisiones judiciales [Anchorage in judicial decision making]. Psicothema, 14, 39-46. Recuperado de: http://www.psicothema.com/pdf/684.pdf

*Farris, K.L. (2007). A developmental perspective on juvenile sexual offenders: The role of childhood abuse in the development of psychopathology and sex offending behavior (Tesis doctoral, Universidad de Auburn). Recuperado de: https://search.proquest.com/docview/622055060?accountid=14542

Fazel, S., Doll, H. y Långström, N. (2008). Mental disorders among adolescents in juvenile detention and correctional facilities: A systematic review and metaregression analysis of 25 surveys. Journal of the American Academy of Child and Adolescent Psychiatry, 47 (9), 1.010-1.019. http://dx.doi.org/10.1097/CHI.ObO13e31817eecf3

Fernández Martínez, R., Kokoulina, E., Campos Mouriño, X., Carballido Araújo, E., García Fuertes, I., Rey Pousada, A. y Vázquez Batán, P. (2018). Ecofenotipos en la depresión mayor: El papel del maltrato físico en la infancia. Revista de la Asociación Española de Neuropsiquiatría, 38 (133), 75-97. http://dx.doi.org/10.4321/S0211-57352018000100004

Férriz, L., Sobral, J. y Gómez-Fraguela, J.A. (2018). Empatía y delincuencia juvenil: Un metaanálisis sobre la relación. Revista Iberoamericana de Psicología y Salud, 9 (1), 1-16. http://dx.doi.org/10.23923/j.rips.2018.01.011

Ford, J.D., Grasso, D.J., Hawke, J. y Chapman, J.F. (2013). Poly-victimization among juvenile justice-involved youths. Child Abuse \& Neglect, 37 (10), 788-800. http://dx.doi.org/10.1016/j.chiabu.2013.01.005

Forouzan, E. y Nicholls, T.L. (2015). Childhood and adolescent characteristics of women with high versus low psychopathy scores: Examining developmental precursors to the malignant personality disorder. Journal of Criminal Justice, 43 (4), 307-320. 
http://dx.doi.org/10.1016/j.jcrimjus.2015.06.001

Fox, B.H., Perez, N., Cass, E., Baglivio, M.T. y Epps, N. (2015). Trauma changes everything: Examining the relationship between adverse childhood experiences and serious, violent and chronic juvenile offenders. Child Abuse \& Neglect, 46, 163-173. http://dx.doi.org/10.1016/j.chiabu.2015.01.011

Foy, D.W., Ritchie, I.K. y Conway, A.H. (2012). Trauma exposure, posttraumatic stress, and comorbidities in female adolescent offenders: Findings and implications from recent studies. European Journal of Psychotraumatology, 3 (1), 17.247. http://dx.doi.org/10.3402/ejpt.v3i0.17247

Frewen, P., Zhu, J. y Lanius, R. (2019). Lifetime traumatic stressors and adverse childhood experiences uniquely predict concurrent PTSD, complex PTSD, and dissociative subtype of PTSD symptoms whereas recent adult non-traumatic stressors do not: results from an online survey study. European Journal of Psychotraumatology, 10 (1), 1-10. http://dx.doi.org/10.1080/20008198.2019.1606625

*Gaete, J., Labbé, N., del Villar, P., Allende, C., Araya, R. y Valenzuela, E. (2018). Mental health and associated factors among young offenders in Chile: A cross-sectional study. Criminal Behaviour and Mental Health, 28 (1), 13-27. http://dx.doi.org/10.1002/cbm.2029

*Ghazali, S.R., Chen, Y.Y. y Aziz, H.A. (2018). Childhood maltreatment and symptoms of PTSD and depression among delinquent adolescents in Malaysia. Journal of Child and Adolescent Trauma, 11 (2), 151-158. http://dx.doi.org/10.1007/s40653-017-0196-2

*Goodkind, S., Ruffolo, M.C., Bybee, D. y Sarri, R. (2009). Coping as a mediator of the supports on depression among girls in juvenile justice. Youth Violence and Juvenile Justice, 7 (2), 100-118. http://dx.doi.org/10.1177/1541204008327140

*Gover, A.R. (2003). Child maltreatment and adjustment to juvenile correctional institutions. Criminal Justice and Behavior, 30 (3), 374-396. http://dx.doi.org/10.1177/0093854803252362

*Gover, A.R. (2004). Childhood sexual abuse, gender, and depression among incarcerated youth. International Journal of Offender Therapy and Comparative Criminology, 48 (6), 683-696. http://dx.doi.org/10.1177/0306624X04264459

*Hill, S.A., Brodrick, P., Doherty, A., Lolley, J., Wallington, F. y White, O. (2014). Characteristics of female patients admitted to an adolescent secure forensic psychiatric hospital. The Journal of Forensic Psychiatry \& Psychology, 25 (5), 503-519. http://dx.doi.org/10.1080/14789949.2014.933863

Ho, S.M.Y., Wai Tong Dai, D., Mak, C. y Wing Kei Liu, K. (2018). Cognitive factors associated with depression and anxiety in adolescents: A two-year longitudinal study. International Journal of Clinical and Health Psychology, $18 \quad$ (3), 227-234. http://dx.doi.org/10.1016/j.ijchp.2018.04.001

*Hoeve, M., Colins, O.F., Mulder, E.A., Loeber, R., Stams, G.J.J.M. y Vermeiren, R.R. J.M. (2015). The association between childhood maltreatment, mental health problems, and aggression in justice-involved boys. Aggressive Behavior, 41 (5), 488-501. http://dx.doi.org/10.1002/ab.21586

*Hunter, J.A., Figuered, A.J., Malamuth, N.M. y Becker, J.V. (2003). Juvenile sex offenders: Toward the development of a typology. Sexual Abuse: A Journal of Reserach and Treatment, 15 (1), 27-48. http://dx.doi.org/10.1177/107906320301500103

Hyun, M., Friedman, S.D. y Dunner, D.L. (2000). Relationship of childhood physical and sexual abuse to adult bipolar disorder. Bipolar Disorders, 2 (2), 131-135. http://dx.doi.org/10.1034/j.1399-5618.2000.020206.x

Instituto Nacional de Estadística, INE (2017). Menores condenados según sexo, edad y nacionalidad. Recuperado de: $h t t p: / / w w w . i n e . e s / j a x i T 3 / D a t o s . h t m ? t=25721$

*Jencks, J.W. y Leibowitz, G.S. (2018). The impact of types and extent of trauma on depressive affect among Male Juvenile sexual offenders. International Journal of Offender Therapy and Comparative Criminology, 62 (5), 1.143-1.163. 
http://dx.doi.org/10.1177/0306624X16676099

Jiménez, T.I. y Estévez, E. (2017). School aggression in adolescence: Examining the role of individual, family and school variables. International Journal of Clinical and Health Psychology, 17 (3), 251-260. http://dx.doi.org/10.1016/j.ijchp.2017.07.002

*Kerig, P.K. y Modrowski, C.A. (2018). Testing gender-differentiated models of the mechanisms linking polyvictimization and youth offending: Numbing and callousness versus dissociation and borderline traits. Journal of Trauma and Dissociation, 19 (3), 347-361. http://dx.doi.org/10.1080/15299732.2018.1441355

*King, D.C., Abram, K.M., Romero, E.G., Washburn, J.J., Welty, L.J. y Teplin, L.A. (2011). Childhood maltreatment and psychiatric disorders among detained youths. Psychiatric Services, 62 (12), 1.430-1.438. http://dx.doi.org/10.1176/appi.ps.004412010

*Lansing, A.E., Plante, W.Y., Beck, A.N. y Ellenberg, M. (2018). Loss and grief among persistently delinquent youth: The contribution of adversity indicators and psychopathyspectrum traits to broadband internalizing and externalizing psychopathology. Journal of Child \& Adolescent Trauma, 11 (8), 1-15. http://dx.doi.org/10.1007/s40653-018-0209-9

*Leduc, K.L. (2002). Alexithymia, trauma, and posttraumatic stress disorder in incarcerated juvenile offenders (Tesis doctoral). EE.UU.: Universidad de California, Fresno. Recuperado de: https://search.proquest.com/docview/619945977?accountid $=14542$

*Lemos, I. y Faísca, L. (2015). Psychosocial adversity, delinquent pathway and internalizing psychopathology in juvenile male offenders. International Journal of Law and Psychiatry, 42-43, 49-57. http://dx.doi.org/10.1016/j.ijlp.2015.08.007

*Lindberg, N., Oshukova, S., Miettunen, J. y Kaltiala-Heino, R. (2016). Do seriously offending girls differ from their age- and offence type-matched male counterparts on psychopathic traits or psychopathy-related background variables? Child and Adolescent Psychiatry and Mental Health, 10 (1), 1-10. http://dx.doi.org/10.1186/s13034-016-0128-1

Llorca-Mestre, A., Malonda-Vidal, E. y Samper-García, P. (2017). Prosocial reasoning and emotions in young offenders and non-offenders. European Journal of Psychology Applied to Legal Context, 9 (2), 65-73. http://dx.doi.org/10.1016/j.ejpal.2017.01.001

López-Pérez, M.G. (2017). Acoso escolar y cibernético en estudiantes universitarios. Revista de Investigación en Educación, 15 (1), 11-26. Recuperado de: http://reined.webs.uvigo.es/index.php/reined/article/view/200/197

*Matsuura, N., Hashimoto, T. y Toichi, M. (2013). Associations among adverse childhood experiences, aggression, depression, and self-esteem in serious female juvenile offenders in Japan. Journal of Forensic Psychiatry and Psychology, 24 (1), 111-127. http://dx.doi.org/10.1080/14789949.2012.746384

*Mcpeek, J.F. (1997). The role of family functioning, coping response style, abuse or trauma and gender; an exploratory study of court-placed adolescen (Tesis doctoral, Universidad de Detroit). Recuperado de: https://search.proquest.com/docview/619258291?accountid=14542

Monteiro, A., Vázquez, M.J., Seijo, D. y Arce, R. (2018). ¿Son los criterios de realidad válidos para clasificar y discernir entre memorias de hechos auto-experimentados y de eventos vistos en vídeo? [Are the reality criteria valid to classify and to discriminate between memories of self-experienced events and memories of video-observed events?]. Revista Iberoamericana de Psicología y Salud, 9 (2), 149-160.

https://doi.org/10.23923/j.rips.2018.02.020

*Moore, T.L. (2003). Sexually victimized and non-sexually victimized adolescent sexual offenders: One group or two? (Tesis doctoral, Universidad Internacional Alliant). Recuperado de: https://search.proquest.com/docview/620621911 ?accountid $=14542$

*Moore, E., Gaskin, C. y Indig, D. (2013). Childhood maltreatment and post-traumatic stress disorder among incarcerated young offenders. Child Abuse \& Neglect, 37 (10), 861-870. http://dx.doi.org/10.1016/j.chiabu.2013.07.012 
*Morais, H.B., Alexander, A.A., Fix, R.L. y Burkhart, B.R. (2018). Childhood sexual abuse in adolescents adjudicated for sexual offenses: Mental health consequences and sexual offending behaviors. Sexual Abuse: Journal of Research and Treatment, 30 (1), 23-42. http://dx.doi.org/10.1177/1079063215625224

Moyano, N., Monge, F.S. y Sierra, J.C. (2017). Predictors of sexual aggression in adolescents: Gender dominance vs. rape supportive attitudes. European Journal of Psychology Applied to Legal Context, 9 (1), 25-31. http://dx.doi.org/10.1016/j.ejpal.2016.06.001

*Nowakowski-Sims, E. y Rowe, A. (2017). The relationship between childhood adversity, attachment, and internalizing behaviors in a diversion program for child-to-mother violence. Child Abuse \& Neglect, 72, 266-275. http://dx.doi.org/10.1016/j.chiabu.2017.08.015

Ogrinc, G., Davies, L., Goodman, D., Batalden, P., Davidoff, F. y Stevens, D. (2015). SQUIRE 2.0 (Standards for QUality Improvement Reporting Excellence): Revised publication guidelines from a detailed consensus process. The Journal of Continuing Education in Nursing, 46 (11), 501-507. http://dx.doi.org/10.3928/00220124-20151020-02

Organización Mundial de la Salud. (2015). Desarrollo en la adolescencia. Recuperado de: https://www.who.int/maternal_child_adolescent/topics/adolescence/dev/es/

Organización Mundial de la Salud. (2016). Maltrato infantil. Recuperado de: https://www.who.int/es/news-room/fact-sheets/detail/child-maltreatment

Perestelo-Pérez, L. (2013). Standards on how to develop and report systematic reviews in Psychology and Health. International Journal of Clinical and Health Psychology, 13 (1), 49-57. Recuperado de: https://www.elsevier.es/en-revista-international-journal-clinicalhealth-psychology-355-pdf-X1697260013849757

*Perez, N.M., Jennings, W.G. y Baglivio, M.T. (2018). A path to serious, violent, chronic delinquency: The harmful aftermath of adverse childhood experiences. Crime and Delinquency, 64 (1), 3-25. http://dx.doi.org/10.1177/0011128716684806

Putnam, F.W. (2003). Ten-year research update review: Child sexual abuse. Journal of the American Academy of Child \& Adolescent Psychiatry, 42 (3), 269-278. http://dx.doi.org/10.1097/00004583-200303000-00006

*Roe-Sepowitz, D. y Krysik, J. (2008). Examining the sexual offenses of female juveniles: The relevance of childhood maltreatment. American Journal of Orthopsychiatry, 78 (4), 405 412. http://dx.doi.org/10.1037/a0014310

*Ručević, S. y Ajduković, M. (2016). Problem behaviors, childhood victimization, and attachment quality in Croatian sample of community and referred psychopathic-like adolescents. Criminal Justice and Behavior, 43 (5), 564-582. http://dx.doi.org/10.1177/0093854815605526

*Ruffolo, M.C., Sarri, R. y Goodkind, S. (2004). Study of delinquent, diverted, and high-risk adolescent girls: Implications for mental health intervention. Social Work Research, 28 (4), 237-245. http://dx.doi.org/10.1093/swr/28.4.237

Schimmenti, A., Di Carlo, G., Passanisi, A. y Caretti, V. (2015). Abuse in childhood and psychopathic traits in a sample of violent offenders. Psychological Trauma: Theory, Research, Practice, and Policy, 7 (4), 340-347. http://dx.doi.org/10.1037/tra0000023

*Silva, T.C., Graña, J.L. y González-Cieza, L. (2014). Self-reported physical and emotional abuse among youth offenders and their association with internalizing and externalizing psychopathology: A preliminary study. International Journal of Offender Therapy and Comparative Criminology, 58 (5), 590-606. http://dx.doi.org/10.1177/0306624X12474975

Stimmel, M.A., Cruise, K.R., Ford, J.D. y Weiss, R.A. (2014). Trauma exposure, posttraumatic stress disorder symptomatology, and aggression in male juvenile offenders. Psychological Trauma: Theory, Research, Practice, and Policy, 6 (2), 184-191. http://dx.doi.org/10.1037/a0032509 
*Taylor, J., James, L.M., Reeves, M.D. y Kistner, J.A. (2009). Borderline personality traits are associated with poor clinical and psychosocial functioning in delinquent boys. Journal of Psychopathology and Behavioral Assessment, 31 (2), 94-103. http://dx.doi.org/10.1007/s10862-008-9097-x

Teplin, L.A., Abram, K.M., McClelland, G.M., Dulcan, M.K. y Mericle, A.A. (2002). Psychiatric disorders in youth in juvenile detention. Archives of General Psychiatry, 59 (12), 11331143. Recuperado de: https://www.ncbi.nlm.nih.gov/pmc/articles/PMC2861992/

Teplin, L.A., Abram, K.M., McClelland, G.M., Washburn, J.J. y Pikus, A.K. (2005). Detecting mental disorder in juvenile detainees: Who receives services. American Journal of Public Health, 95 (10), 1.773-1.780. http://dx.doi.org/10.2105/AJPH.2005.067819

*Tossone, K., Wheeler, M., Butcher, F. y Kretschmar, J. (2018). The role of sexual abuse in trauma symptoms, delinquent and suicidal behaviors, and criminal justice outcomes among females in a juvenile justice diversion program. Violence against Women, 24 (8), 973-993. http://dx.doi.org/10.1177/1077801217724921

Tuvblad, C., Bezdjian, S., Raine, A. y Baker, L.A. (2013). Psychopathic personality and negative parent-to-child affect: A longitudinal cross-lag twin study. Journal of Crime and Justice, 41 (5), 331-341. http://dx.doi.org/10.1016/j.jcrimjus.2013.07.001

Ulloa Flores, R.E. y Navarro Machuca, I.G. (2011). Estudio descriptivo de la prevalencia y tipos de maltrato en adolescentes con psicopatología. Salud Mental, 34 (3), 219-225. Recuperado de: http://www.scielo.org.mx/pdf/sm/v34n3/v34n3a5.pdf

Urrútia, G. y Bonfill, X. (2010). PRISMA declaration: A proposal to improve the publication of systematic reviews and meta-analyses. Medicina Clínica, 135 (11), 507-511. http://dx.doi.org/10.1016/j.medcli.2010.01.015

*Vahl, P., Colins, O.F., Lodewijks, H.P.B., Lindauer, R., Markus, M.T., Doreleijers, T.A.H. y Vermeiren, R.R. (2016). Psychopathic traits and maltreatment: Relations with aggression and mental health problems in detained boys. International Journal of Law and Psychiatry, 46, 129-136. http://dx.doi.org/10.1016/j.ijlp.2016.02.006

*Vahl, P., Van Damme, L., Doreleijers, T., Vermeiren, R. y Colins, O. (2016). The unique relation of childhood emotional maltreatment with mental health problems among detained male and female adolescents. Child Abuse \& Neglect, 62, 142-150. http://dx.doi.org/10.1016/j.chiabu.2016.10.008

Vallejos, M., Bertone, M.S. y Muniello. J. (2014). Prevalencia de maltrato infantil y trastornos psiquiátricos en una población femenina privada de su libertad. Revista Neuropsicología, Neuropsiquiatría y Neurociencias, 14 (3), 103-113. Recuperado de: https://revistannn.files.wordpress.com/2014/07/12-14-3-vallejo-et-al-_maltrato-infantil-ytrastornos-psiquic3a1tricos.pdf

Vallejos, M. y Bertone, M.S. (2016). Eventos traumáticos en la infancia en una población masculina privada de su libertad: Estudio de prevalencia. Revista de la Facultad de Ciencias Médicas, 73 (2), 75-82. Recuperado de:

https://revistannn.files.wordpress.com/2016/12/10-vallejos-salvador-bertone_eventostraumacc81ticos-en-nincc83os-privados-de-libertad.pdf

Veneziano, C., Veneziano, L. y Legrand, S. (2000). The relationship between adolescent sex offender behaviors and victim characteristics with prior victimization. Journal of Interpersonal Violence, 15 (4), 363-374. http://dx.doi.org/10.1177/088626000015004002

von Elm, E., Altman, D.G., Egger, M., Pocock, S.J., Gøtzsche, P.C. y Vandenbroucke, J.P. (2014). The strengthening the reporting of observational studies in epidemiology (STROBE) statement: Guidelines for reporting observational studies. International Journal of Surgery, 12 (12), 1.495-1.499. http://dx.doi.org/10.1016/j.ijsu.2014.07.013

*Wanklyn, S.G., Day, D.M., Hart, T.A. y Girard, T.A. (2012). Cumulative childhood maltreatment and depression among incarcerated youth: impulsivity and hopelessness as 
potential intervening variables. Child Maltreatment, $17 \quad$ (4), 306-317. http://dx.doi.org/10.1177/1077559512466956

Wolff, K.T., Baglivio, M.T. y Piquero, A.R. (2017). The relationship between adverse childhood experiences and recidivism in a sample of juvenile offenders in community-based treatment. International Journal of Offender Therapy and Comparative Criminology, 61 (11), 1.210-1.242. http://dx.doi.org/10.1177/0306624X15613992

Wolff, N. y Shi, J. (2012). Childhood and adult trauma experiences of incarcerated persons and their relationship to adult behavioral health problems and treatment. International Journal of Environmental Research and Public Health, 9 (5), 1.908-1.926. http://dx.doi.org/10.3390/ijerph9051908

Yugueros-García, A.J. (2013). La delincuencia femenina. Una revisión teórica. FORO. Revista de Ciencias Jurídicas y Sociales, Nueva Época, 16 (2), 311-316. http://dx.doi.org/10.5209/rev_foro.2013.v16.n2.43943

Zahn, M.A., Hawkins, S.R., Chiancone, J. y Whitworth, A. (2008). The girls study groupCharting the way to delinquency prevention for girls. Washington, D.C.: Office of Juvenile Justice and Delinquency Prevention. Recuperado de: https://www.ncjrs.gov/pdffiles1/ojjdp/223434.pdf

Zhang, J. y Zheng, Y. (2018). Childhood maltreatment profiles among incarcerated Chinese males and their associations with personality disorder symptoms and criminal behaviors. Psychiatry Research, 268, 272-278. http://dx.doi.org/10.1016/j.psychres.2018.06.063 